\title{
Challenges in Biology and Medicine with Synchrotron Infrared Light
}

\author{
P. Dumas ${ }^{a}$, L.M. Miller ${ }^{b}$ And M.J. Tobin ${ }^{c}$ \\ ${ }^{a}$ SOLEIL Synchrotron, Saint-Aubin, BP 48, 91192 Gif-sur-Yvette Cedex, France \\ ${ }^{b}$ National Synchrotron Light Source, Brookhaven National Laboratory \\ Upton, NY 11973, USA \\ ${ }^{c}$ Australian Synchrotron Project, 800 Blackburn Road, Clayton Victoria, Australia
}

\begin{abstract}
The brightness (or brilliance) of synchrotron radiation was exploited in infrared microspectrosocopy. Among application of this synchrotron-based microanalytical technique, biological and biomedical investigations, at the diffraction-limited spot size, are exhibit of an increasing interest among almost all the existing infrared beamline worldwide. This paper is presenting the main properties of such a source, coupled with an infrared microscope. Several important applications in biomedical field are reported: cancer cells studies and drug effects, human substantia nigra in Parkinson's disease, $\beta$-amyloids deposits in Alzheimer's disease.
\end{abstract}

PACS numbers: 87.80.Dj, 87.80.-y, 87.64.km, 87.56.B-, 87.19.xj, 87.16.-b, 87.15.bd, 78.30.-j

\section{Introduction}

Microscopic analyses with infrared (IR) spectroscopy, i.e. IR microspectroscopy, are made possible by coupling a modified light microscope with, in most cases, a Fourier transform infrared spectrometer [1]. Infrared microspectroscopy has been widely used over the past $20+$ years for biological and biomedical applications, for revealing the biochemical makeup of numerous cells and tissues $[2,3]$. Infrared spectroscopy forms part of "biophotonics", a field of research that merges biology and optics and covers an ensemble of techniques which exploit light or more generally all forms of photonic energy in the field of life sciences. Infrared spectroscopy may be broadly defined as the study of the absorption characteristics, arising from the molecular motion of materials, upon interaction with a broadband infrared source. Today, it is commonly accepted that the chemical pathology of samples can be characterized by their infrared signatures since vibrational spectroscopy is able to detect subtle biochemical changes within tissues [4-6]. The high heterogeneity of biological sample requires spatially resolved studies, and thus microscopy has become an essential tool for accurate biological and biomedical investigations [7]. The addition of a microscope as an accessory to conventional Fourier transform infrared (FTIR) spectrometers has brought the potential to examine tissues at cellular resolution. It has led to infrared imaging, where biochemical and spatial information are combined. It carries a high level of molecular information, but with a spatial resolution limited by the weakness of the laboratory source (at a scale of several tens of square microns).

IR microspectroscopy has been more widely employed than the Raman microspectroscopy, due to the superior $\mathrm{S} / \mathrm{N}$ obtained, but progress in the Raman microscope is making this technique much more challenging and very complementary to infrared microscopy [8].

In order to improve the spatial resolution, higher brightness infrared sources are required and they do exist (e.g. lasers). However, the most useful source is emitted from synchrotron radiation, as it is a "white" source that delivers the entire infrared spectral range, i.e. from far-IR to near-IR, with an exceptionally high brightness compared to conventional laboratory sources [9].

The specific aims of this article is to familiarize readers with the properties of infrared emission from synchrotron radiation, with the use of synchrotron-based IR microspectroscopy (SR-FTIRM) beamlines, and with the important benefits of SR-FTIRM for biological and biomedical applications. Rather than presenting a comprehensive review of all up-to-date biomedical applications of SR-FTIR spectromicroscopy, which have recently been reviewed [3] we shall focus on contents that illustrate the requirements and utility of SR-FTIRM as a chemical probe for tracking biomolecular changes relevant in biomedicine.

\section{Synchrotron radiation and infrared emission}

\subsection{The making of synchrotron light}

Electron-based synchrotron light sources use magnetic fields to bend the electrons into a closed orbit. Synchrotron radiation (SR) is produced at each of these "bending" magnets. The emitted radiation spans an extremely broad spectral domain, extending from the X-ray regime to the very far infrared region. Infrared radiation is generated by electrons traveling at relativistic velocities, either in a curved path through a constant magnetic field (i.e. bending magnet radiation [10]) or when their trajectories encounter variable magnetic fields, e.g. at the edges of bending magnets (BM) (i.e. edge radiation, (ER)) (Fig. 1). 


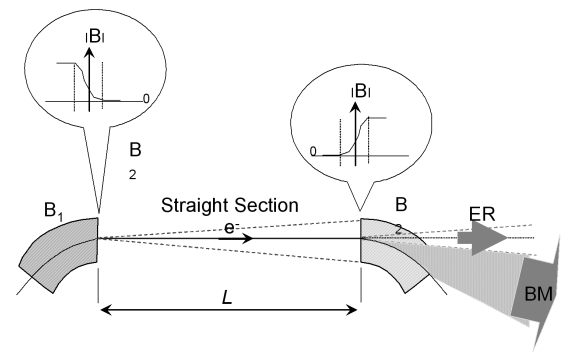

Fig. 1. Schematic drawing of the infrared emission from ER and BM radiation. $L$ is the length of the straight section, and this influences the interference effects with the upstream ER dipole emission.

In the latter, the ER is generated by a high-energy charged particle when it passes the entrance or exits through the region of a rapid change of magnetic field amplitude of a bending magnet. The photons emitted at two adjacent bending magnets bounding a straight section, appear in the same cone and are subsequently synchronized by the electron itself. This leads to an interference effect, which manifests itself in oscillations of radiation intensity $[11,12]$.

Flux and brightness for the two types of infrared emission are almost equivalent, but the opening angle of the edge radiation is narrower than that of the SR from constant field of a bending magnet. The intensity profiles of the two types of emission are also different, and also depend upon wavelength. In Fig. 2 the distribution profiles at two wavelengths $(10 \mu \mathrm{m}$ and $100 \mu \mathrm{m})$ have been calculated for a medium energy storage ring $(3.0 \mathrm{GeV})$ for a prototypical opening angle of the dipole chamber of $20 \times 40 \mathrm{mrad}$ for the collection of bending magnet infrared emission, and $20 \times 20 \mathrm{mrad}$ for the collection of the edge radiation infrared emission. These profiles illustrate that, for bending magnet emission, the angle of emission is larger than that of the edge radiation.

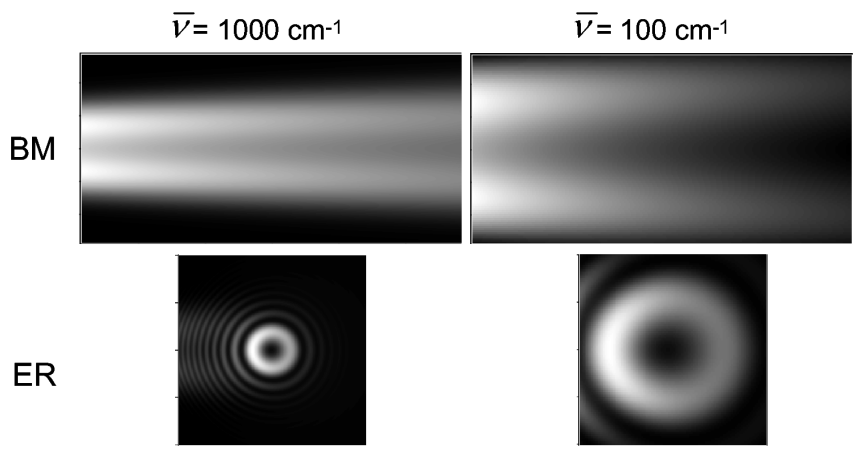

Fig. 2. Intensity distribution of edge radiation, for two wavelengths $(\lambda=100 \mu \mathrm{m}$ (left column) and $\lambda=10 \mu \mathrm{m}$ (right column)). The distribution has been calculated for a BM emission, and an ER emission. This calculation has been carried out for the case of a storage ring of $3 \mathrm{GeV}$, and a vertical aperture of $20 \mathrm{mrad}$.
This has some advantages for engineering purposes, since the dipole chamber exit port in a synchrotron storage ring has to be enlarged for bending magnet radiation. Thus today, both sources are used for extraction, depending upon availability of a straight section and/or bending magnet.

A marked difference between the two sources is their polarization properties (Fig. 3).

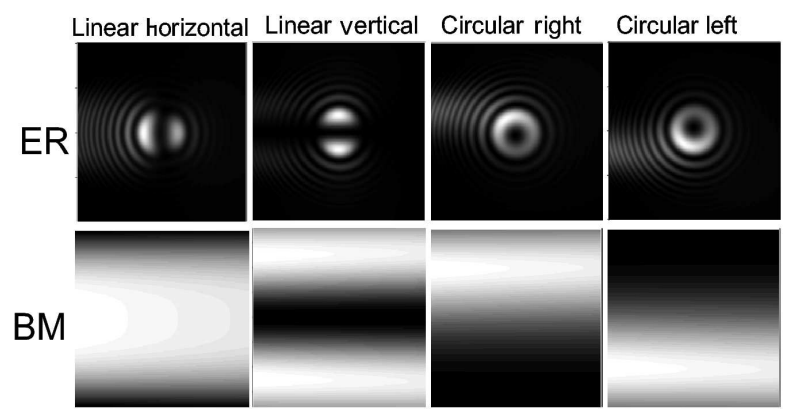

Fig. 3. Intensity distribution for various polarization states for ER and BM radiation.

Compared to bending magnet emission, which is strictly horizontally polarized in the plane of the electron trajectory, edge radiation has a radial polarization. Circular right and left polarization, as well as vertical polarization, can be selected by collecting the appropriate portion of the infrared beam emission, as seen in Fig. 3.

\subsection{Flux versus brightness}

The brightness of synchrotron light is defined as the photon flux or power emitted per source area and solid angle. Most of the experiments in synchrotron infrared spectroscopy exploit the brightness advantage of the source, while the flux advantage is exploited mainly in the far infrared region. It is important to note that synchrotron radiation does not provide a much higher photon flux than a conventional IR source (such as a globar), unlike the traditional case in the X-ray region. In fact, the total flux can be much smaller (about one to two orders of magnitude) in the mid infrared region, although it becomes superior at long wavelengths (Fig. 4).

The crucial factor is that the effective synchrotron source size is quite small, i.e. on the order of $\approx 100 \mu \mathrm{m}$ or less for newer synchrotron storage rings. Thus, the light is emitted into a narrow range of angles, and the resulting brightness is vastly increased.

As an example, one can estimate the value of the brightness (also called brilliance or spectral radiance) to be

$$
B(\lambda)=\frac{F_{s r c}}{\sigma_{x} \sigma_{y} \sigma_{x}^{\prime} \sigma_{y}^{\prime}},
$$

where $F_{s r c}$ is the photon flux, $\sigma_{x}$ and $\sigma_{y}$ are the rms dimensions of the photon beam in $x$ and $y$ directions respectively, and $\sigma_{x}^{\prime}$ and $\sigma_{y}^{\prime}$ are the emission angles, taken at the photon beam waist. 


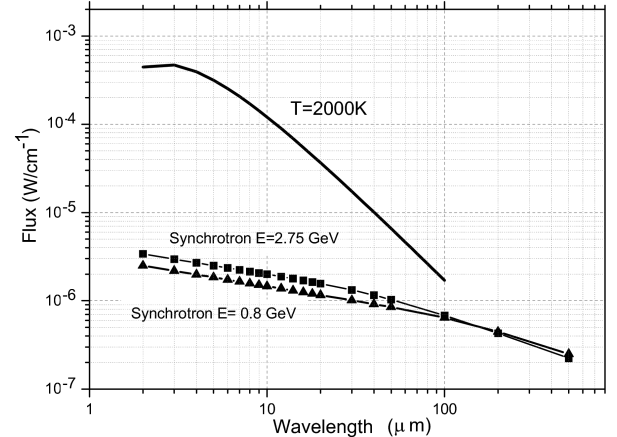

Fig. 4. Comparison of the infrared flux, expressed in $\mathrm{W} / \mathrm{cm}^{-1}$, between a blackbody heated up to $2000 \mathrm{~K}$, and a synchrotron emission, such as NSLS (0.85 GeV nominal energy) or SOLEIL (2.75 GeV nominal energy).

Following this equation, one can calculate the source brightness ratio between the synchrotron source and the blackbody source.

$$
\begin{aligned}
& \frac{B_{\text {synchrotron }}(\lambda)}{B_{\text {blackbody }}(\lambda)} \\
& =\frac{\left(F_{\text {src }}\right)_{\text {synchrotron }}(\phi)}{\left(F_{\text {src }}\right)_{\text {blackbody }}(\phi)} \frac{\left(\sigma_{x} \sigma_{y} \sigma_{x}^{\prime} \sigma_{y}^{\prime}\right)_{\text {blackbody }}}{\left(\sigma_{x} \sigma_{y} \sigma_{x}^{\prime} \sigma_{y}^{\prime}\right)_{\text {synchrotron }}} \\
& \approx 2 \times 10^{2} .
\end{aligned}
$$

Thus, the apparent brightness of synchrotron radiation can be 2-3 orders of magnitude higher than that of a globar source. However, it is interesting to note that, contrary to X-ray irradiation, sample heating is negligible, permitting analysis of single cells for timescales from hours to days [13].

Infrared emission from a synchrotron light source was proposed many years ago as a useful source for throughput-limited IR experiments [10, 14-17]. The initial applications of synchrotron IR light focused on long wavelength applications (i.e. often called the far-IR or $\mathrm{THz}$ range) and were carried out at Tantalus, Daresbury and Bessy I, starting in the 1970's [18]. The real boost in infrared activities at synchrotron facilities started shortly thereafter, at UVSOR [19], NSLS [20-22], and later LURE SuperACO [23]. All beamlines were designed with reasonably large apertures in order to efficiently extract the infrared photons. However, at this time, electron beam motions in the synchrotron ring generated very noisy photon beams. In addition, the beam current, which is the primary factor that determines IR intensity, was quite limited. Most science programs utilizing such infrared beamlines concentrated on surface science and solid state physics [24, 25]. However, synchrotron-based infrared applications exploded with the marriage of synchrotron IR light and the infrared microscope, where the brightness advantage of synchrotron IR light was demonstrated for microanalysis [26, 27].

The high brightness of infrared synchrotron radiation has impacted the field of infrared microspectroscopy in a variety of scientific disciplines, including hard and soft condensed matter [26, 28], geology [29], and biology . The principal motivation for synchrotron-based IR microspectroscopy is to achieve significantly greater lateral resolution (typically at the diffraction limit) while recording data of superior signal-to-noise characteristics without resorting to prohibitively long acquisition times.

Accordingly, facilities for performing IR spectroscopy have expanded throughout the world in response to an increasing demand for beamtime from the scientific community. In North America, the NSLS, New York presently operates five IR microscopes, while IR microscopes can also be found at the ALS, Berkeley and CAMD, Baton Rouge and SRC, Stoughton (USA); the CLS, Saskatoon (Canada); in Asia, UVSOR, Okasaki and SPring8, Nishi-Harima (Japan); NSRRC, Hsinchu (Taiwan) operate IR microscopes. In Europe, IR activities exist at the SRS, Daresbury (UK); ESRF, Grenoble (France); MAXLAB, Lund (Sweden); Da $\phi$ ne, Frascati (Italy); ELETTRA, Trieste (Italy); ANKA, Karlsruhe (Germany); BESSY II, Berlin (Germany), and SOLEIL, Paris (France). The Australian Synchrotron, Melbourne and the Swiss Light Source (Villigen - Switzerland) also have a new IR program opened to users.

Other facilities that are planning IR spectroscopy programs include Diamond, Rutherford Lab (UK); DELTA, Dortmund (Germany); Metrology Light Source (MLS) (Berlin, Germany), ALBA (Barcelona, Spain), NSRC (Thailand), SESAME (Jordan), the Pohang Accelerator Laboratory (Korea), NSRC (Thailand), INDUS1 (India) and NSRL, Hefei (China).

\section{Infrared microscopes and spatial resolution}

When considering the available spatial resolution, two issues are important to take into account. The first is the acceptable signal-to-noise $(\mathrm{S} / \mathrm{N})$ of the data. The flux - and thus $\mathrm{S} / \mathrm{N}$ - decreases drastically as apertures in the IR microscope are reduced to confine the IR beam to smaller areas. The second issue is diffraction (residual optical aberrations of the focusing optical elements are neglected). The resolution issue has been evaluated both theoretically and experimentally by Carr [35]. With the use of a synchrotron source, the diffraction limit is achieved when the microscope's apertures define a region with dimensions equal to the wavelength of interest. However, the use of confocal optical arrangement leads to a $30 \%$ improvement in the spatial resolution, in agreement with diffraction theory [35].

Infrared microscopes are commercially available from a number of companies worldwide. In recent years, these microscopes have been improved so that they now include many of the features of research-grade optical microscopes such as polarization, Nomarski measurements, difference interference contrast (DIC), and epifluorescence. They are also equipped with sophisticated software for generating and analyzing chemical images.

Typically, IR microscopes are configured in one of two ways - for FTIR microspectroscopy (FTIRM) or FTIR 
imaging (FTIRI). In a few cases, single IR microscopes can operate in both configurations. For an FTIRM instrument, a small area (a "point") is spectroscopically sampled by the instrument, and an image is built up by raster-scanning the specimen through the focused beam. Since only a single point is sampled at a time, these instruments use a single-element detector. The microscope uses reflecting Schwarzschild-type objectives to avoid absorption and chromatic aberrations over the large mid-IR spectral range. One objective serves to focus the light onto the specimen, while the other collects the light and relays it onto the detector. An aperture is used to constrain the illuminated or detected area on the specimen.

When examining the chemical makeup of biological cells and tissues with an IR microscope, it is important to achieve sub-cellular spatial resolution. For both FTIRM and FTIRI, the spatial resolution is limited by the wavelengths of IR light, which are longer than visible light wavelengths used for conventional optical microscopy. The diffraction-limited spatial resolution is dependent upon the wavelength of light and the numerical aperture (NA) of the focusing optic [36]. Typical IR microscopes utilize the Schwarzschild objectives with a NA of $\approx 0.6$. Some microscopes utilize a single aperture before the sample, which controls the region illuminated. With a single aperture, the diffraction-limited spatial resolution is approximately $2 \lambda / 3$ [35]. Thus for the mid-IR range, the diffraction-limited spatial resolution is approximately from $1.7 \mu \mathrm{m}$ (at $4000 \mathrm{~cm}^{-1}$ ) to $13 \mu \mathrm{m}\left(\right.$ at $500 \mathrm{~cm}^{-1}$ ). Other microscopes operate in a confocal arrangement, where a second aperture is placed after the sample to define the region being sensed by the IR detector. For such a confocal microscope, where objectives and apertures are placed both before and after the sample, the spatial resolution is improved to $\sim \lambda / 2$ [35]. In addition, the confocal arrangement also reduces the Schwarzschild first- and higher-order diffraction rings, dramatically improving image contrast [35].

For these microscopes, very few modifications are needed in order to adapt the commercial instrument to a synchrotron infrared source. The synchrotron beam is collimated as it enters the interferometer, which modulates the wavelength of light, and then is directed towards the IR microscope.

\subsection{Confocal geometry and contrast fidelity}

Due to their complexity and heterogeneity, analysis of biomedical samples benefits greatly from high quality data collected at high spatial resolution. As such, domain sizes close to the diffraction limit are important and blurring effects can distort the spectroscopic information. Since this aspect is a fundamental advantage of the infrared synchrotron source, the following illustrated the importance of preserving the contrast fidelity.

As an example of the importance of a confocal geometry, Fig. 5 shows a ring-shaped test pattern with a $14 \mu \mathrm{m}$ ring diameter and a thickness of $2 \mu \mathrm{m}$. The center and right images represent the calculated chem- ical image with a $6 \mu \mathrm{m}$ wavelength for a single aperture (non-confocal geometry) and two apertures (confocal geometry), respectively. It is very clear that, for the non-confocal geometry, one may conclude that there is a chemical component with a characteristic frequency at $6 \mu \mathrm{m}$. This shows that an artifact can be introduced for high spatial resolution. (a)

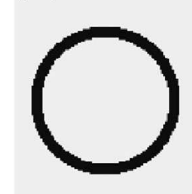

(b)

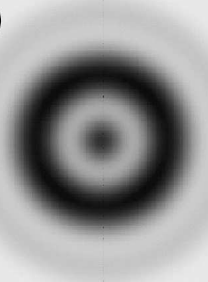

(c)

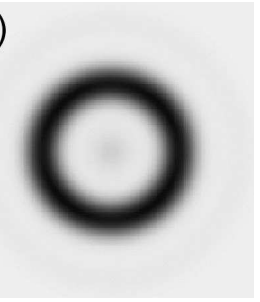

Fig. 5. Calculated images for a $14 \mu \mathrm{m}$ diameter ring-shaped object at $\lambda=6 \mu \mathrm{m}$ : (a) actual object; (b) image for a (single aperture) non-confocal $32 \times$ Schwarzschild objective with $\mathrm{NA}=0.65$; (c) image for a confocal (dual aperture) $32 \times$ Schwarzschild objective with NA $=0.65$ (from [60]).

To emphasize this point, we have recorded the chemical image of lipids (at $2920 \mathrm{~cm}^{-1}$ ) for a cross-section of human hair using either a confocal synchrotron IR microscope or a thermal source-based microscope equipped with an array detector. In both case, the projected size of the aperture was approximately $\approx 6 \times 6 \mu \mathrm{m}^{2}$. Marked differences between the two images can be observed in Fig. 6.

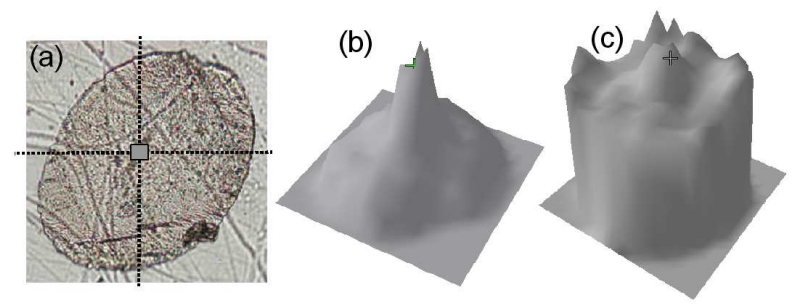

Fig. 6. Chemical image of the lipid distribution throughout a cross-section of a human hair. The analysis was performed on the same section (a) with a microscope employing an internal source and an array detector (b), and with a synchrotron-powered infrared microscope with a single detector element (c).

First, the lipids content appears to be much higher with the conventional microscope equipped with array detector (Fig. 6b). Second, and more importantly, the presence of lipids in the outside layer of the hair section, the cuticle, is not revealed with a non-confocal arrangement.

The two above examples illustrate the main advantage of using the synchrotron source in biological specimens: one can preserve the contrast fidelity at very high spatial resolution, while maintaining a high spectral quality (i.e. 
a high signal-to-noise ratio), which are both essential for detecting subtle biochemical changes in cells and tissues.

\subsection{Spectral quality improvements using synchrotron infrared microscopy}

In order to illustrate the superior spectral quality (signal to noise), while importantly preserving the contrast fidelity, spectra obtained on the same individual cell (HELA cell), using either the synchrotron beam, or the internal source, with an aperture of $6 \times 6 \mu \mathrm{m}^{2}$, are displayed in Fig. 7.

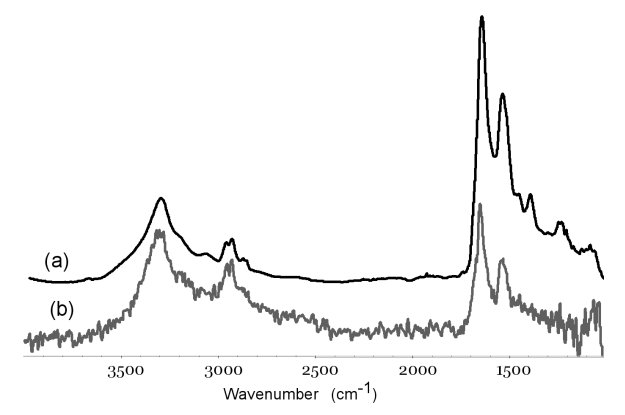

Fig. 7. Infrared spectra recorded with a dual aperture of $6 \times 6 \mu \mathrm{m}^{2}$, at $4 \mathrm{~cm}^{-1}$ resolution, coadding 128 scans with (a) the synchrotron source and (b) with the internal (globar) source.

\section{Biological and biomedical studies using synchrotron infrared microscopy}

Infrared microspectroscopy provides a direct indication of a sample's biochemistry [3]. Armed with information on sample histology and pathology, variations in nucleic acid, protein, and lipid content or structure, can assess chemistry variations of diseased states. Specifically, the dominant absorption features in lipid spectra are found in the region from 2800 to $3000 \mathrm{~cm}^{-1}$, and are assigned to the asymmetric and symmetric $\mathrm{C}-\mathrm{H}$ stretching vibrations of $\mathrm{CH}_{3}\left(2956\right.$ and $\left.2874 \mathrm{~cm}^{-1}\right)$ and $\mathrm{CH}_{2}$ (2922 and $\left.2852 \mathrm{~cm}^{-1}\right)$. In addition, strong bands at $1736 \mathrm{~cm}^{-1}$ arise from ester $\mathrm{C}=\mathrm{O}$ groups in the lipid. Protein spectra have two primary features, the amide I (1600-1700 $\left.\mathrm{cm}^{-1}\right)$ and amide II (1500-1560 $\left.\mathrm{cm}^{-1}\right)$ bands, which arise primarily from the $\mathrm{C}=\mathrm{O}$ and $\mathrm{C}-\mathrm{N}$ stretching vibrations of the peptide backbone, respectively. The frequency of the amide I band is particularly sensitive to protein secondary structure [37-39]. In nucleic acids, the region in the range $1000-1500 \mathrm{~cm}^{-1}$ contains contributions from asymmetric $\left(1224 \mathrm{~cm}^{-1}\right)$ and symmetric $\left(1087 \mathrm{~cm}^{-1}\right) \mathrm{PO}^{2-}$ stretching vibrations.

\subsection{Sub-cellular analysis of cancerous cells}

Cancer screening of tissues using conventional infrared microscopy is an active area of ongoing research [40]. Complementarily, synchrotron infrared studies provide unique information for diagnosis of the states of single cells. This is clearly exemplified in the following study of a drug interaction with individual cancer cells [41].

The following example is taken from the study of Photodynamic therapy of cancerous cells [41].

Photodynamic therapy (PDT) is a technique employing a photosensitizer, that, after penetration into cells, exhibits anticancer activity when irradiated with light of wavelength matching its main absorption band [41]. In this example, HELA cells were studied interacting with hypocrellin A (HA), a lipid-soluble peryloquinone derivative isolated from natural fungus sacs of Hypocrella bambusae. HA exhibits an important absorption band at around $460 \mathrm{~nm}$. HELA cells incubated with various concentration of HA, and irradiated at $460 \mathrm{~nm}$ during variable exposure time, were studied using MTT assays (rapid colorimetric assay for cellular growth and survival). The lowest rate of survival was found for an incubation in $10 \mu \mathrm{M}$ of HA solution, and subsequent illumination with a light dose of $48 \mathrm{~J} \mathrm{~cm}^{-2}$ [41].

Several individual cells were probed with synchrotron infrared microscopy, while varying the probed area from $3 \times 3 \mu \mathrm{m}^{2}$ up to $12 \times 12 \mu \mathrm{m}^{2}$. The experiments were performed at the infrared beamline at ESRF (ID21-IR) and at SOLEIL (SMIS beamline).

Figure 8 displays the spectra recorded from the same HELA cell at various aperture sizes. It is evident that the amide I band exhibits an upward frequency shift when the aperture is larger than $6 \times 6 \mu \mathrm{m}^{2}$. This is roughly the average size of the HELA cell nucleus. This behavior can be interpreted as a higher contrast provided by probing subcellular regions containing mainly the nucleus, which requires the confocal geometry and synchrotron IR source. At larger apertures, an averaging of the infrared signal modifies the band position of the Amide I.

The marked change of the amide I band inside the nucleus is related to the specific secondary structure of proteins inside the nucleus and this is consistent with the fact that such a change is often correlated with the cell apoptosis as well as the cell death $[42,43]$.

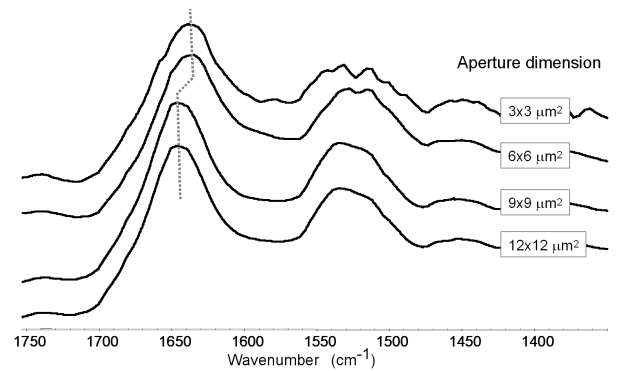

Fig. 8. Infrared spectra recorded on the same cell, at different size of the projected aperture centered on the single cell. A marked change in the amide I band shape is evident for apertures below $6 \times 6 \mu \mathrm{m}$. This corresponds to the average size of the nucleus. This shows that the use of confocal geometry, combined with the high brightness of the synchrotron source, reveals the specific secondary structure of proteins inside the cell nucleus. 
Such a behavior is further confirmed by recording spectra across one diameter of one cell, with an aperture of $3 \times 3 \mu \mathrm{m}^{2}$ (Fig. 9).

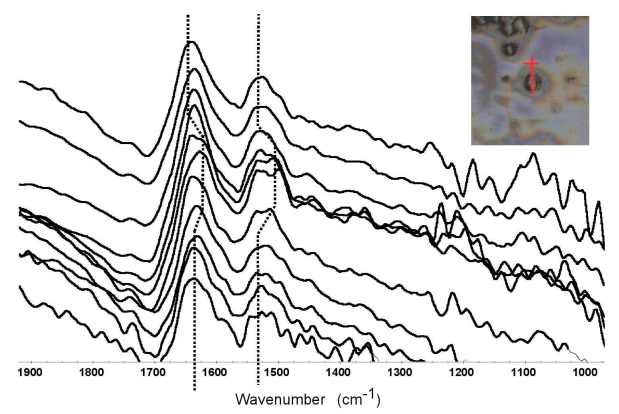

Fig. 9. Linear scan recorded across an individual HELA cell with a $3 \times 3 \mu \mathrm{m}$ square aperture and a step of $3 \mu \mathrm{m}$. Spectra were acquired at $4 \mathrm{~cm}^{-1}$ resolution and with 128 accumulations per spectrum.

The diagnostic capability, for biomedical application, is readily strengthened by using synchrotron infrared microscopy. For HELA cells manifesting a very low rate of survival after HA interaction and light exposure, the biochemical signature of the cell death is well revealed, when a large number of analysis are made either in the cytoplasm or the nucleus (such a differentiation is due to the sub-cellular resolution provided by the synchrotron source).

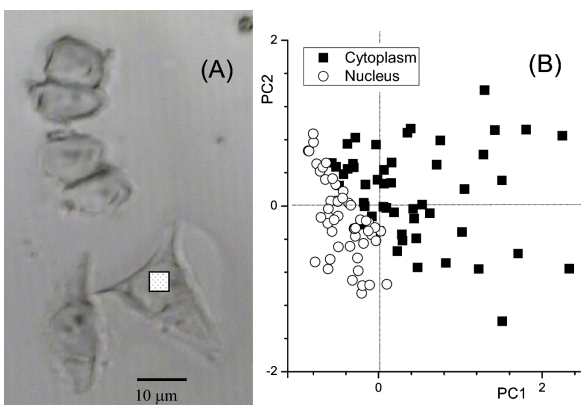

Fig. 10. (A) Probing area inside HELA cells using synchrotron infrared microscopy. The dimension of the projected aperture on the sample was set to $6 \times 6 \mu \mathrm{m}^{2}$ (represented as a dashed square), allowing analysis of either the nucleus or the cytoplasm of each cell. (B) PCA analysis of the whole set of spectra taken inside the nucleus and the cytoplasm of about 100 cells. There is a clear separation of the spectra into two clusters, where the discrimination is $92 \%$ accounted along the PC1 axis.

A large set of infrared spectra, recorded either inside the cytoplasm or the nucleus of about 100 individuals cells (Fig. 10A), have been analyzed using principal component analysis (PCA). The score plot recorded in Fig. 10B, exhibits a clear separation of two classes: spectra recorded inside the cytoplasm and those recorded inside the nucleus. Correspondingly, the loading plot (Fig. 11) show that the spectral variation rely mainly in the secondary structure difference between these two sub-regions of the cells, with a more pronounced $\beta$-type secondary structure inside the nucleus.

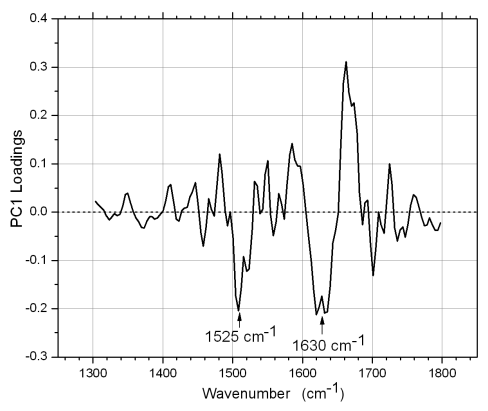

Fig. 11. Loadings plot of PC1, showing that, for spectra lying along the negative values of $\mathrm{PC} 1$ axis, $\beta$-sheets vibrational bands are responsible for the marked loading difference, as manifested by the peaks at 1630 and $1520 \mathrm{~cm}^{-1}$, and are predominantly located inside the nucleus.

The study of drug effect (in this case the light interaction with cancer cells incubed by HA), has been carried out with high spectral contrast using the synchrotron source [41].

Other studies have shown that spectral differences exist between normal and cancerous oral epithelial cells $[44,45]$ healthy and nutrient-repleted Micrasterias hardyi algal cells [46] and HepG2 cells exposed to low doses of 2,3,7,8-tetrachlorodibenzo- $p$-dioxin [13]. Variations in DNA/RNA content and packing have also been demonstrated during the cell cycle of human lung epithelial cells [42] as well as in prostate cancer cells [47]. Individual mouse hybridoma B cells have been examined during necrosis and the end phases of mitosis [48], and also during the process of apoptosis [49].

\subsection{Biomolecular investigation of human substantia nigra (SN) in Parkinson's disease}

SR-FTIR microscopy was used to distinguish chemical composition and morphology of the human substantia nigra (SN) of brain between normal and Parkinson's diseased tissues [50]. The Parkinson disease (PD) is a chronic, progressive neurodegenerative movement disorder. It results from the degeneration and loss of dopamine-producing nerve cells in the brain, mainly in the SN [51]. It is well accepted that PD is a multietiological disease. Most researchers believe that oxidative stress and mitochondrial dysfunction play a crucial role in degeneration and death of nerve cells in the SN. Moreover, dyshomeostasis of Fe ions, protein aggregation, increased peroxidation of lipids, changes in the activity of enzymes of the antioxidant system and some other factors are considered at investigations of PD pathogenesis. The aforementioned processes should induce changes in main biological molecules.

Therefore, this region was investigated with a high spatial resolution, thanks to the synchrotron source, in or- 
der to detect any variation in the biochemical composition of the human substantia nigra of brain between normal and Parkinson's diseased tissues. The experiments were carried out at the IR beamline MIRAGE, at LURE (France), by scientists from Faculty of Physics and Applied Computer Science, AGH University of Science and Technology, Kraków and Institute of Neurology, Jagiellonian University Medical College, Kraków, Poland [3].

Thin tissue sections were examined, focusing more particularly on nerve cell bodies that are affected in PD. The major spectral differences between normal (control) and PD tissues appear at the following vibrational frequencies: 2930, 2850, 1655, 1380, 1236, 1173 and $1086 \mathrm{~cm}^{-1}$, as well as in the amide I (secondary structure fingerprints of the proteins) region (Fig. 12A, B).

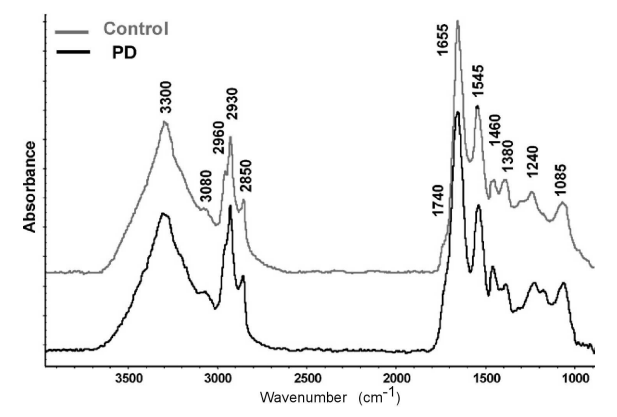

Fig. 12. The comparison of FTIR absorption spectra acquired in the neurons of substantia nigra for the control and Parkinson's diseased (PD) cases. Spectra were collected with a $6 \times 6 \mu \mathrm{m}^{2}$ aperture.

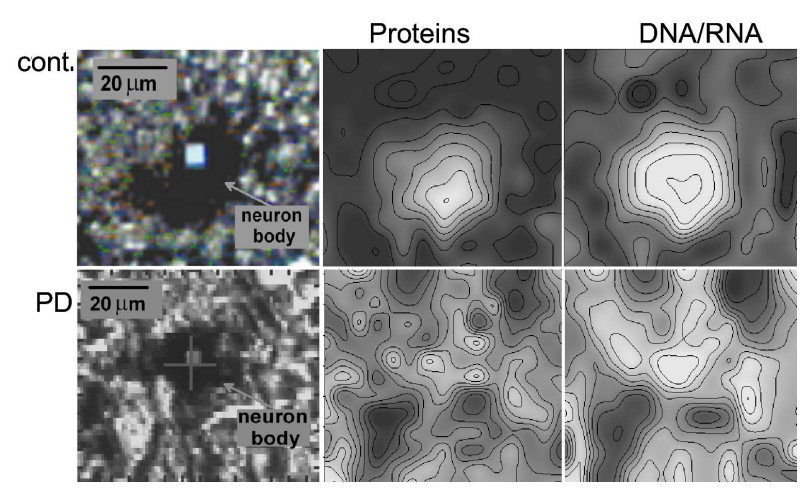

Fig. 13. Chemicals images of protein and DNA/RNA obtained with a $6 \times 6 \mu \mathrm{m}^{2}$ aperture, using a synchrotron infrared microscope. Spectra have been collected in PD and control SN neurons [50].

The infrared imaging of the protein and nucleic acids functional groups indicates a higher concentration of these two components inside the neuron cell body, while this is markedly not the case for PD samples (Fig. 13).

These results strengthen the hypothesis that PD is a multietiological disorder. Moreover, the reported results clearly indicate that, in addition to a distinct visual observation, the diseased nerve cells exhibits change of their biochemical composition. It suggests that disturbances of normal functioning of SN neurons appear before their morphological atrophy.

\subsection{SR-FTIR study of $\beta$-amyloid deposits in Alzheimer's disease}

Alzheimer's disease (AD) is characterized by the misfolding and plaque-like accumulation of a naturally occurring peptide in the brain called amyloid beta $(\mathrm{A} \beta)$. This process has been associated with the binding of metal ions such as iron $(\mathrm{Fe})$, copper $(\mathrm{Cu})$, and zinc ( $\mathrm{Zn)}$ [52]. It is thought that metal dyshomeostasis is involved in protein misfolding and may lead to oxidative stress and neuronal damage.

However, the exact role of the misfolded proteins and metal ions in the degenerative process of $\mathrm{AD}$ is not yet clear. Synchrotron infrared microspectroscopy has been used to image the in situ secondary structure of amyloid plaques in brain tissue of $\mathrm{AD}$ patients with high spatial resolution. Figure 14B illustrates the FTIR spectra obtained inside the plaque (Fig. 14A). Some brain samples have been shown to exhibit additional absorption features in the FTIRM spectra, assigned to creatine (Fig. 14C) [53]. Chemical images of $\mathrm{A} \beta$ and creatine distribution from a hippocampal section of Alzheimer diseased human brain, obtained with an aperture of $3 \times 3 \mu \mathrm{m}$ and $1 \mu \mathrm{m}$ step size, are displayed in Fig. 14D and E, respectively.

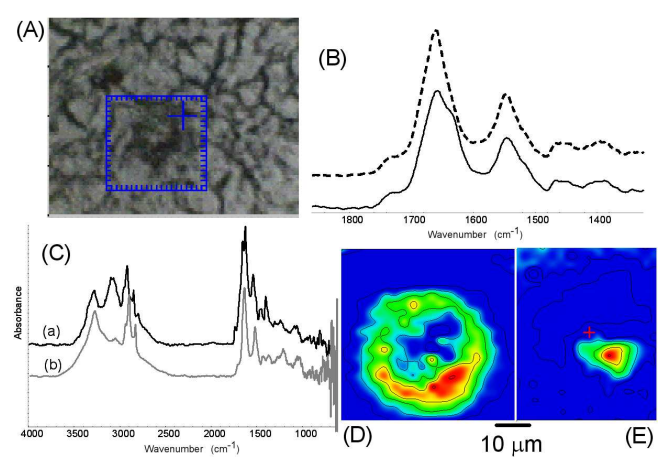

Fig. 14. (A) Light microscope image of Alzheimer's diseased brain tissue. (B) Infrared spectra recorded inside and outside the plaque, showing the shoulder at $1630 \mathrm{~cm}^{-1}$, assigned to $\beta$-amyloid. (C) Infrared spectra from inside the plaque. One of them displays additional absorption features, which are assigned to creatine. (D) Infrared image of amyloid plaque distribution, calculated as a peak height ratio of $1630 / 1655 \mathrm{~cm}^{-1}$. (E) Chemical image of creatine distribution inside the plaque.

Synchrotron X-ray fluorescence (SXRF) microprobe is a complementary technique used to probe trace elements with sensitivities in the sub $\mathrm{mg} \mathrm{kg}^{-1}$ range and a spatial resolution similar to FTIRM $(2-10 \mu \mathrm{m})$ [54]. Because of the low power deposition that X-rays provide and the ability to conduct the measurements in air, these analyses can be done non-destructively on a much wider array of 
sample types, especially relatively fragile biological samples. For example, the alterations in trace metals such as $\mathrm{Fe}, \mathrm{Cu}$, and $\mathrm{Zn}$ have been observed in neurological diseases such as Parkinson's disease, amylotrophic lateral sclerosis, Alzheimer's disease, and prion diseases [55-57].

It would be very beneficial if both FTIRM and SXRF information from a single sample can be combined. However, this requires precise overlap of the IR and X-ray images. This is an additional potentiality provided by the use of a synchrotron beam and has been successfully achieved at the National Synchrotron Light Source at Brookhaven National Laboratory [58]. FTIRM and SXRF microprobe have been used to correlate protein structure and metal ion accumulation, respectively, in amyloid plaques from brain tissue of $\mathrm{AD}$ patients. FTIRM is used to image the protein secondary structure in $\mathrm{AD}$ tissue, where amyloid plaques are known to be associated with elevated levels of $\beta$-sheet content $[55,59]$.

\section{Outlook and conclusions}

Nowadays, the efforts devoted to the stability of the electron beam in storage rings, as well as for the isolation of vibrational noise in beamline design, result in a very stable synchrotron infrared source. The high brightness can, therefore, be exploited for very rapid analyses of single cells, for chemical screening or cell dynamics. It might even be possible to couple this approach with optical tweezers.

One of the pitfalls of FTIRM remains the diffraction limited spot size. Any effort in improving the resolution will be extremely beneficial. Two-dimensional detectors, and a sophisticated treatment for mathematical deconvolution of the data (i.e. point spread function deconvolution) has been recently suggested [60].

Synchrotron infrared spectroscopy is very attractive for many scientific disciplines, in a wavelength energy domain extending from the very far- to the near-infrared. The activity will continue to grow, thanks to the high demand from the user community. Several synchrotron facilities are planning, designing, or building new infrared beamlines.

One unique direction for the future, which is starting to be exploited at synchrotron facilities, is the opportunity to combine infrared microscopy with other synchrotron based techniques. The strategy for efficient combination relies upon the choice of adequate sample substrates, and good coordination between beamtime allocations at different end stations. Other combinatory approaches are underway using synchrotron-based (auto-) fluorescence microscopy and infrared microscopy at SOLEIL synchrotron.

The Raman microscopy is also an excellent approach to vibrational spectroscopy, with an equivalent, or often better, spatial resolution than synchrotron infrared microscopy. The data are complementary, but often Raman does not provide adequate spectral quality (i.e. signal to noise), data can be complicated by intrinsic fluores- cence emission, and samples can suffer from laser damage. Thus, it is valuable to combine the two microspectroscopic approaches, and this should be made available for users at synchrotron facilities.

Several improvements are now being proposed for synchrotron infrared microscopy. Nowadays, focal plane array detectors are being implemented in microscopes that use a globar source, and the performance of these detectors has not yet being exploited with a synchrotron source. Clearly, the size of the detector array has to be adapted to match the projected size of the synchrotron beam, in order to keep the brightness advantage of this source. These small detector arrays might be well available soon, and will allow faster acquisition data, and a slightly improved lateral resolution using point-spread function (PSF) deconvolution.

\section{Acknowledgments}

The authors are grateful to S. Srichan, F. Jamme, and C. Sandt (SOLEIL), G.L. Carr, R. Smith, A. Kretlow, M. Ruppel, A. Leskovjan (NSLS), G.P. Williams (Jefferson Labs) for their very active collaboration and interesting discussions.

\section{References}

[1] J.M. Chalmers, P.R. Griffiths, Theory and Instrumentation, Wiley, New York 2002.

[2] D.L. Wetzel, S.M. LeVine, Science 285, 1224 (1999).

[3] L.M. Miller, P. Dumas, Biochim. Biophys. Acta 1758, 846 (2006).

[4] M. Jackson, H.H. Mantsch, in: Infrared Spectroscopy of Biomolecules, Wiley-Liss, 1996, p. 311.

[5] M. Jackson, J.R. Mansfield, B. Dolenko, R.L. Somorjai, H.H. Mantsch, P.H. Watson, Cancer Det. Prev. 23, 245 (1999).

[6] H.H. Mantsch, L.-P. Choo-Smith, R.A. Shaw, Vibrat. Spectrosc. 30, 31 (2002).

[7] S. Boydston-White, T. Gopen, S. Houser, J. Bargonetti, M. Diem, Appl. Spectrosc. 53, 148A (1999).

[8] G.J. Puppels, T.C. Bakker Schut, P.J. Caspers, In vivo Raman spectroscopy, in Handbook of Raman Spectroscopy, Eds. I.R. Lewis, H.G.M. Edwards, Dekker, New York 2002, p. 549.

[9] W. Duncan, G.P. Williams, Appl. Opt. 22, 2914 (1983).

[10] W. Duncan, G.P. Williams, Appl. Opt. 22, 2914 (1983).

[11] R.A. Bosch, Nucl. Instrum. Methods Phys. Res. A 386, 525 (1997).

[12] Y.L. Matthis, P. Roy, B. Tremblay, A. Nucara, S. Lupi, P. Calvani, A. Gerschel, Phys. Rev. Lett. 80, 1220 (1998)

[13] H.Y.N. Holman, R. Goth-Goldstein, M.C. Martin, M.L. Russel, W.R. McKinney, Environ. Sci. Technol. 34, 2513 (2000).

[14] D. Einfeld, J. Nagel, BESSY Report No TB34, 1981.

[15] P. Lagarde, Infrared Phys. 18, 395 (1979). 
[16] J.R. Stevenson, H. Ellis, R. Bartlett, Appl. Opt. 12, 2884 (1973).

[17] J.R. Stevenson, J.J. Lathcart, Nucl. Instrum. Methods Phys. Res. B 172, 367 (1980).

[18] E. Schweizer, J. Nagel, W. Braun, E. Lippert, A.M. Bradshaw, Nucl. Instrum. Methods Phys. Res. A 239, 630 (1985).

[19] T. Nanba, Int. J. Millimeter Waves 7, 759 (1986).

[20] G.P. Williams, Nucl. Instrum. Methods Phys. Res. A 291, 8 (1990)

[21] G.P. Williams, C.J. Hirschmugl, E.M. Kneedler, E.A.Sullivan, D.P. Siddons, Y.J. Chabal, F. Hoffmann, K.D. Moeller, Rev. Sci. Instrum. 60, 2176 (1989).

[22] G.P. Williams, P.Z. Takacs, R.W. Klaffky, M. Schleifer, Nucl. Instrum. Methods Phys. Res. A 246, 165 (1986).

[23] P. Roy, Y.-L. Mathis, S. Lupi, A. Nucara, B. Tremblay, A. Gerschel, Synchrotron Radiation News 8, 10 (1995).

[24] L. Forro, G.L. Carr, G.P. Williams, D. Mandrus, L. Mihaly, Phys. Rev. Lett. 65, 1941 (1990).

[25] C.J. Hirschmugl, G.P. Williams, F.M. Hoffmann, Y.J. Chabal, Phys. Rev. Lett. 65, 408 (1990).

[26] G.L. Carr, M. Hanfland, G.P. Williams, Rev. Sci. Instrum. 66, 1643 (1995).

[27] G.L. Carr, J. Reffner, G.P. Williams, Rev. Sci. Instrum. 66, 1490 (1995).

[28] G. Ellis, M.A. Gómez, C. Marco, J. Macromol. Sci., Part B, Phys. 43, 191 (2004).

[29] N. Guilhaumou, P. Dumas, G.L. Carr, G.P. Williams, Appl. Spectrosc. 52, 1029 (1998).

[30] P. Dumas, L. Miller, J. Biol. Phys. 29, 201 (2003).

[31] N. Jamin, P. Dumas, J. Moncuit, W.H. Fridman, J.L. Teillaud, G.L. Carr, G.P. Williams, Proc. Natl. Acad. Sci. 95, 4837 (1998)

[32] L.M. Miller, C.S. Carlson, G.L. Carr, M.R. Chance, Cell. Mol. Biol. 44, 117 (1998).

[33] L.M. Miller, G.L. Carr, M. Jackson, P. Dumas, G.P. Williams, Synchrotron. Radiat. News 13, 31 (2000).

[34] L.M. Miller, P. Dumas, N. Jamin, J.L. Teillaud, J. Miklossy, L. Forro, Rev. Sci. Instrum. 73, 1357 (2002).

[35] G.L. Carr, Rev. Sci. Instrum. 72, 1613 (2001).

[36] R.G. Messerschmidt, in: Practical Guide to Infrared Microspectroscopy, Ed. H. Humecki, Dekker, 1995, p. 3.

[37] D.M. Byler, H. Susi, Biopolymers 25, 469 (1986).

[38] H. Susi, D.M. Byler, Biochem. Biophys. Res. Commun. 115, 391 (1983).

[39] H. Susi, D.M. Byler, J.M. Purcell, J. Biochem. Biophys. Methods 11, 235 (1985).

[40] M.J. Walsh, M.J. German, M. Singh, H.M. Pollock, A. Hammiche, M. Kyrgiou, H.F. Stringfellow, E. Paraskevaidis, P.L. Martin-Hirsch, F.L. Martin, Cancer Lett. 246, 1 (2007).
[41] S. Chio-Srichana, M. Refregiers, F. Jamme, S. Kascakovac, S. Deshayes, V. Rouam, P. Dumas, Biochim Biophys. Acta, accepted for publication.

[42] H.Y.N. Holman, D.L. Perry, M.C. Martin, W.R. McKinney, Biopolymers 57, 329 (2000).

[43] J. Sulé-Suso, D. Skingsley, G.D. Sockalinghum, A. Kohler, G. Kegelaer, M. Manfait, A.El Haj, Vibrat. Spectrosc. 38, 179 (2005).

[44] M.J. Tobin, M.A. Chesters, J.M. Chalmers, F.J.M. Rutten, S.E. Fisher, I.M. Symonds, A. Hitchcock, R. Allibone, S. Dias-Gunasekara, Faraday Discuss. 126, 27 (2004).

[45] M. Diem, L. Chiriboga, P. Lasch, A. Pacifico, Biopolym. Biospectrosc. 67, 349 (2002).

[46] P. Heraud, B.R. Wood, M.J. Tobin, J. Beardall, D. McNaughton, FEMS Microbiol. Lett. 249, 219 (2005).

[47] E. Gazi, J. Dwyer, N.P. Lockyer, J. Miyan, P. Gardner, C.A. Hart, M.D. Brown, N.W. Clarke, Vibrat. Spectrosc. 38, 193 (2005).

[48] N. Jamin, P. Dumas, J. Moncuit, W.H. Fridman, J.L. Teillaud, G.L. Carr, G.P. Williams, Cell. Mol Biol. 44, 9 (1998).

[49] N. Jamin, L.M. Miller, J. Moncuit, W.H. Fridman, P. Dumas, J.L. Teillaud, Biopolymers 72, 366 (2003).

[50] M. Szczerbowska-Boruchowska, P. Dumas, M.Z. Kastyak, J. Chwiej, M. Lankosz, D. Adamek, A. Krygowska-Wajs, Arch. Biochem. Biophys. 459, 241 (2007).

[51] E.E. Kolesnikova, T.V. Serebrovskaya, Neurophysiology 35, 56 (2003).

[52] M.A. Lovell, J.D. Robertson, W.J. Teesdale, J.L. Campbell, W.R. Markesbery, J. Neurol. Sci. 158, 47 (1998).

[53] M. Rak, M.R. Del Bigio, S. Mai, D. Westaway, K.M. Gough, Biopolymers 4, 201 (2007).

[54] S.R. Sutton, P.M. Bertsch, M. Newville, M. Rivers, A. Lanzirotti, P. Eng, Rev. Mineral. Geochem. 49, 429 (2002).

[55] L.M. Miller, Q. Wang, T.P. Telivala, R.J. Smith A. Lanzirotti, J. Miklossy, J. Struct. Biol. 155, 30 (2006).

[56] B. Tomik, J. Chwiej, M. Szczerbowska-Boruchowska M. Lankosz, S. Wojcik, D. Adamek, G. Falkenberg, S. Bohic, A. Simionovici, Z. Stegowski, A.Szczudlik, Neurochem. Res. 31, 321 (2006).

[57] J.F. Collingwood, A. Mikhaylova, M.Davidson, C.Batich, W.J. Streit, J. Terry, J. Dobson, J. Alzheimers Dis. 7, 267 (2005).

[58] L.M. Miller, Q. Wang, R.J. Smith, H. Zhong, D. Elliott, J. Warren, Analyt. Bioanalyt. Chem. 387, 1705 (2007).

[59] Q. Wang, A. Kretlow, M. Beekes, D. Naumann, L.M. Miller, Vibrat. Spectrosc. 38, 61 (2005).

[60] G.L. Carr, O. Chubar, P. Dumas, in: Spectrochemical Analysis Using Infrared Detectors, Ed. I.W.L.R. Bahrgava, Blackwell, 2006. 